\title{
Clinicopathologic and prognostic significance of C-reactive protein/albumin ratio in patients with solid tumors: an updated systemic review and meta-analysis
}

\author{
Jiayuan $\mathrm{Wu}^{1}$, Wenkai Tan ${ }^{2}$, Lin Chen ${ }^{3}$, Zhe Huang ${ }^{4}$ and Shao Mai ${ }^{2}$ \\ ${ }^{1}$ Clinical Research Center, The Affiliated Hospital of Guangdong Medical University, Zhanjiang 524001, Guangdong Province, \\ PR China \\ ${ }^{2}$ Department of Gastroenterology, The Affiliated Hospital of Guangdong Medical University, Zhanjiang 524001, Guangdong \\ Province, PR China \\ ${ }^{3}$ Department of Cardiac Surgery, The Affiliated Hospital of Guangdong Medical University, Zhanjiang 524001, Guangdong \\ Province, PR China \\ ${ }^{4}$ Department of Gastrointestinal Surgery, The Affiliated Hospital of Guangdong Medical University, Zhanjiang 524001, \\ Guangdong Province, PR China
}

Correspondence to: Jiayuan $\mathrm{Wu}$, email: 87537665@qq.com

Keywords: C-reactive protein/albumin ratio; prognosis; clinicopathological features; solid tumors; undated meta-analysis Received: September 15, 2017

Accepted: December 05, 2017

Published: January 11, 2018

Copyright: Wu et al. This is an open-access article distributed under the terms of the Creative Commons Attribution License 3.0 (CC BY 3.0), which permits unrestricted use, distribution, and reproduction in any medium, provided the original author and source are credited.

\section{ABSTRACT}

C-reactive protein/albumin ratio (CAR) was originally used as a novel inflammation-based prognostic score in predicting outcomes in septic patients. Recently, more and more studies have reported the prognostic value of pretreatment CAR in solid tumors. However, the results remain controversial rather than conclusive. We conducted a meta-analysis based on 24 studies with 10203 patients to explore the relationship between CAR and survival outcomes in patients with solid tumors. The correlation between CAR and clinicopathological parameters was also assessed. Hazard ratio (HR) or odds ratio (OR) with its $95 \%$ confidence interval (CI) was applied to be the effect size estimate. The overall results showed that elevated CAR was associated with shorter overall survival (OS) (including 23 studies and 10067 patients) and poorer disease-free survival (DFS) (including 6 studies and 2904 patients). Significant associations between high CAR level and poor OS were also found in the subgroup analyses of study region, cancer type, primary treatment, clinical stage, cut-off selection, sample size, and cut-off value. Moreover, subgroup analyses demonstrated that study region, primary treatment, clinical stage, sample size, and cut-off value did not alter the prognostic value of CAR for DFS. Furthermore, elevated CAR was correlated with certain phenotypes of tumor aggressiveness, such as poor histological grade, serious clinical stage, advanced tumor depth, positive lymph node metastasis, and positive distant metastasis. Together, our meta-analysis suggests that elevated level of serum CAR predicts worse survival and unfavorable clinical characteristics in cancer patients, and CAR may serve as an effective prognostic factor for solid tumors.

\section{INTRODUCTION}

In 1863, Rudolf Virchow first provided the hypothesis of a possible correlation between inflammation and malignant tumor according to the presence of leukocytes within tumors [1]. Yet, it is only during the last twenty years that clear evidence has been obtained that inflammation plays a decisive role in carcinogenesis and tumor progression; for example, inflammation regulates tumor behavior at different stages of tumor development, including initiation, promotion, malignant conversion, invasion, and metastasis [2]. Probable mechanisms 
might be that inflammation could result in malnutrition, immune dysfunction, up-regulation of growth factors, and angiogenesis. Lately, epidemiological studies have revealed that infection-related inflammation contributes to approximately $20 \%$ of all cancer cases worldwide, and chronic inflammation increases the risk of human cancers of almost all organs/tissues, which predisposes individuals to various types of cancers [3]. Several inflammation-inducible factors, such as air pollution, foreign bodies and ultraviolet radiation, are also associated with carcinogenesis [4]. In some cases, inflammatory cells can help to form a tumor microenvironment, which is an indispensable actor in the neoplastic process. Moreover, increasing evidences have proved that anti-inflammatory therapy can reduce the risk and prolong the survival of patients in some cancer types, such as ovarian cancer, cervical cancer, and gastrointestinal malignances, which further supports the close connection between inflammation and cancer development $[5,6]$. Thus, the understanding of cancer-related inflammation could provide novel and alternative strategies for biological intervention of malignant tumors. On the other hand, malnutrition is common in patients with solid tumors, especially in progressive stage. Malnutrition can weaken a number of defense mechanisms in human body, including physiologic barrier, immune system, and phagocyte function. This problem has been proved to be correlated with increased susceptibility to infection, poor curative response, serious therapeutic side effects, and worse survival [7]. Several studies have showed that nutritional interventions can help cancer patients to maintain body weight, improved quality of life, and decrease mortality [8, 9]. Therefore, it is critical to precisely and early identify nutritional risk in cancer patients.

It is a consensus that a pretreatment and effective parameter to evaluate survival probability and prognosis is necessary for decision-making concerning clinical therapy of malignant tumors. Traditionally, the tumornodes-metastasis (TNM) staging system has been the most common tool to predict clinical outcomes and to formulate rational treatment strategies for patients with solid tumors [10]. Nevertheless, patients with the same clinical stage often present large variations in clinical outcomes, especially in some cases with advanced disease. Moreover, TNM staging does not fully consider many essential variables, including the biological variability of the tumor itself, patients' characteristics, laboratory test, and treatment approach, thus, it is inaccurate for predicting prognosis [11]. Except the tumor stage, some other histopathological indicators have been reported to be oncological prognostic indicators by previous studies, such as tumor size, histologic grade, and vascular or nodal involvement, but these variables can only be evaluated after surgical exploration [12]. Therefore, it is urgent to look for reliable prognostic markers for better risk stratification and optimal therapeutic plans of cancer patients.
Given the close relationship between inflammation and cancer, several inflammation- based prognostic systems have been developed to predict the clinical outcome during the course from bench to bedside. These factors, including C-reactive protein (CRP), Glasgow Prognostic Score (GPS), modified Glasgow Prognostic Score (mGPS), high-sensitivity modified Glasgow prognostic score (HS-mGPS), neutrophil to lymphocyte ratio (NLR), platelet to lymphocyte ratio (PLR), and systemic immune-inflammation index (SII), are all easily obtainable from peripheral blood samples, and have been validated in many types of cancer [13-15]. Additionally, not only the physical and metabolic effects of the disease but also the effects of anticancer treatment can lead to inadequate food intake, decreased physical activity, and catabolic metabolic derangement, resulting in cancer patients at risk of malnutrition [16]. Serum albumin (ALB) level is closely related to the degree of malnutrition, so it is commonly used as an indicator of nutritional status. Furthermore, numerous studies have suggested that the lower the level of serum ALB, the worse the prognosis of cancer patients $[17,18]$. Thus, assessment of serum ALB has emerged as a potential prognostic factor in various cancers, since nutritional status can be corrected prior to therapy.

The C-reactive protein/albumin ratio (CAR), represented as a combination of serum CRP and ALB counts, was initially used as a new inflammation-based prognostic score for the purpose of predicting mortality in patients with sepsis [19]. CAR indicates the balance of the inflammatory and nutritional status, also making it a useful index for predicting prognosis in malignance. Recently, numerous studies reported that elevated CAR level was associated with poor prognosis in solid tumors. However, some studies revealed conflicting findings due to the variance in study design, sample size and patient feature. Although a meta-analysis on this topic has been published, only 10 publications were included in that meta-analysis [20]. There have been more than 14 papers continuously published since this meta-analysis was conducted. Thus, the real value of CAR in predicting prognosis of solid tumors has not yet been fully elucidated. For these reasons, we embarked on this update meta-analysis to derive a more precise estimation of the prognostic impact of CAR in patients with solid tumors.

\section{RESULTS}

\section{Description of included studies}

The process of literature search was shown in Figure 1. Initially, 106 papers were generated in the primary electronic search in the major databases. Twentyfour full-text articles published from 2015 to 2017 met the inclusion criteria and were selected for our metaanalysis [21-44]. A total of 10203 patients diagnosed 
with various cancers, including laryngeal squamous cell carcinoma (LSCC) [22], ovarian cancer (OC) [23], renal cell carcinoma (RCC) [24], non-small cell lung cancer (NSCLC) [25], pancreatic cancer (PC) [26-29], colorectal cancer (CRC) [30-33], nasopharyngeal cancer (NPC) [21, 34-36], gastric cancer (GC) [37, 42], oral squamous cell carcinoma (OSCC) [38], hepatocellular carcinoma (HCC) [39, 43], esophageal squamous cell carcinoma (ESCC) [40, 41], small cell lung cancer (SCLC) [44], were included. These studies originated from China [21-24, 27, 28, 33-36, 39-42, 44], Japan [26, 30-32, 37, 43], and Korea [25, 29, 38], respectively. Twenty-three articles reported the outcomes of overall survival (OS) [21-31, 33-44], and 6 studies presented disease free survival (DFS) as survival endpoint [22, 24, 26, 32, 34, 37]. Seventeen studies included cancer patients with all disease stages (Stage I-IV or limited- extensive stage) [22-26, 28-31, 34-36, 38, 39, 41, 43, 44], three studies recruited patients with Stage I-III [37, $40,42]$, and four studies reported advanced stages (III, IV, or III-IV) [21, 27, 32, 33]. The primary treatments were extremely various among these 24 included studies, including surgery [22-24, 26, 31, 37-43], chemotherapy (CT) $[21,25,27,29,30,32,33,44]$, and concurrent chemoradiotherapy (CCRT) [34-36]. The hazard ratios (HRs) and the corresponding 95\% confidence intervals (CIs) of these 24 studies were all directly extracted from the outcomes of multivariate analysis. The cut-off values of defining elevated CAR were calculated by the receiver operating characteristic (ROC) curve in 19 studies [22$24,26,28-35,37-43]$, by cutoff finder in 4 studies [21, $25,27,44]$, and by median value in 1 study [36], which ranged from 0.03 to 0.68 , and were extremely different among the including studies. According to the quality

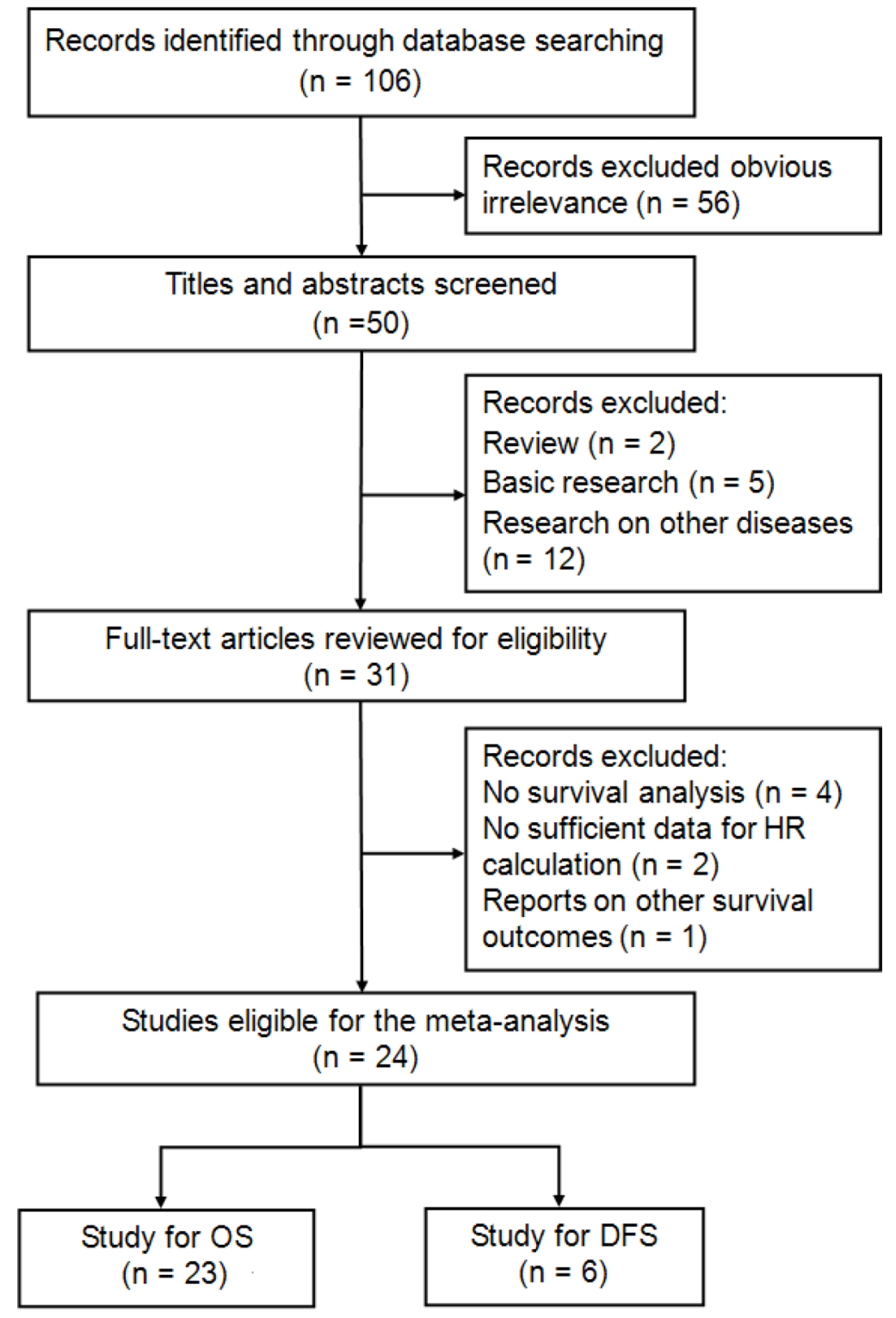

Figure 1: Flow diagram of the study selection process and specific reasons for exclusion in the meta-analysis. 
Table 1: Pooled and subgroup analyses of the main results for the meta-analysis of overall survival

\begin{tabular}{|c|c|c|c|c|c|c|c|}
\hline \multirow{2}{*}{ Categories } & \multirow{2}{*}{$n$} & \multirow{2}{*}{ Model } & \multirow{2}{*}{ HR (95\% CI) } & \multirow{2}{*}{$Z$} & \multirow{2}{*}{$P$} & \multicolumn{2}{|c|}{ Heterogeneity } \\
\hline & & & & & & $I^{2}$ & $\boldsymbol{P}_{\mathrm{h}}$ \\
\hline Overall survival (OS) & $23(10067)$ & Random & $1.95(1.70-2.25)$ & 9.33 & $<0.001$ & $68.9 \%$ & $<0.001$ \\
\hline \multicolumn{8}{|l|}{ Study region } \\
\hline China & $15(8371)$ & Random & $1.84(1.57-2.17)$ & 7.41 & $<0.001$ & $74.2 \%$ & $<0.001$ \\
\hline Japan & $5(1409)$ & Fixed & $1.72(1.60-1.85)$ & 6.85 & $<0.001$ & $0.6 \%$ & 0.403 \\
\hline Korea & $3(287)$ & Random & $2.39(1.34-4.24)$ & 2.96 & 0.003 & $50.9 \%$ & 0.131 \\
\hline \multicolumn{8}{|l|}{ Cancer type } \\
\hline $\mathrm{PC}$ & $4(814)$ & Random & $2.25(1.52-3.34)$ & 4.04 & $<0.001$ & $69.8 \%$ & 0.019 \\
\hline $\mathrm{CRC}$ & $3(874)$ & Fixed & $2.26(1.71-2.99)$ & 5.70 & $<0.001$ & $0.0 \%$ & 0.678 \\
\hline NPC & $4(4814)$ & Fixed & $1.50(1.29-1.74)$ & 5.32 & $<0.001$ & $37.1 \%$ & 0.189 \\
\hline ESCC & $2(891)$ & Random & $1.84(1.06-3.20)$ & 2.18 & 0.030 & $85.6 \%$ & 0.008 \\
\hline GC & $2(839)$ & Fixed & $1.73(1.31-2.29)$ & 3.87 & $<0.001$ & $0.0 \%$ & 0.390 \\
\hline $\mathrm{HCC}$ & $2(364)$ & Fixed & $2.73(2.07-3.60)$ & 7.12 & $<0.001$ & $0.0 \%$ & 0.352 \\
\hline $\mathrm{HNC}$ & $2(169)$ & Fixed & $3.49(1.78-6.85)$ & 3.63 & $<0.001$ & $31.3 \%$ & 0.228 \\
\hline Others & $4(1302)$ & Fixed & $1.41(1.24-1.60)$ & 5.27 & $<0.001$ & $44.2 \%$ & 0.146 \\
\hline \multicolumn{8}{|l|}{ Primary treatment } \\
\hline Surgery & $12(3773)$ & Random & $2.06(1.66-2.55)$ & 6.60 & $<0.001$ & $71.2 \%$ & $<0.001$ \\
\hline $\mathrm{CT}$ & $7(1242)$ & Random & $2.05(1.49-2.81)$ & 4.42 & $<0.001$ & $71.4 \%$ & 0.002 \\
\hline CCRT & $3(4666)$ & Random & $1.53(1.20-1.94)$ & 3.43 & 0.001 & $50.8 \%$ & 0.131 \\
\hline NR & $1(386)$ & Random & $2.07(1.59-2.70)$ & 5.39 & $<0.001$ & NA & NA \\
\hline \multicolumn{8}{|l|}{ Clinical stage } \\
\hline All & $17(8231)$ & Random & $1.82(1.57-2.12)$ & 7.82 & $<0.001$ & $64.4 \%$ & $<0.001$ \\
\hline I - III & $3(1307)$ & Fixed & $2.04(1.67-2.50)$ & 6.94 & $<0.001$ & $42.9 \%$ & 0.173 \\
\hline Advanced & $3(529)$ & Random & $2.61(1.65-4.13)$ & 4.10 & $<0.001$ & $66.4 \%$ & 0.051 \\
\hline \multicolumn{8}{|l|}{ Cut-off selection } \\
\hline ROC & $18(8745)$ & Random & $1.90(1.63-2.21)$ & 8.37 & $<0.001$ & $65.1 \%$ & $<0.001$ \\
\hline Cut-off finder & $4(913)$ & Random & $2.15(1.27-3.65)$ & 2.86 & 0.004 & $85.1 \%$ & $<0.001$ \\
\hline Median & $1(409)$ & Random & $2.09(1.43-3.05)$ & 3.84 & $<0.001$ & NA & NA \\
\hline \multicolumn{8}{|l|}{ Sample size } \\
\hline$<200$ & $10(1288)$ & Fixed & $2.27(1.93-2.68)$ & 9.87 & $<0.001$ & $0.0 \%$ & 0.459 \\
\hline$\geq 200$ & 13 (8779) & Random & $1.81(1.52-2.15)$ & 6.76 & $<0.001$ & $75.5 \%$ & $<0.001$ \\
\hline \multicolumn{8}{|l|}{ Cut-off value } \\
\hline$<0.1$ & $11(7138)$ & Random & $1.92(1.55-2.37)$ & 6.04 & $<0.001$ & $60.6 \%$ & 0.005 \\
\hline$\geq 0.1$ & $12(2929)$ & Random & $1.98(1.62-2.41)$ & 6.77 & $<0.001$ & $75.4 \%$ & $<0.001$ \\
\hline
\end{tabular}

PC pancreatic cancer; CRC colorectal cancer; NPC nasopharyngeal carcinoma; ESCC esophageal squamous cell carcinoma; GC gastric cancer; HCC hepatocellular carcinoma; HNC head and neck cancer (including laryngeal squamous cell carcinoma and oral squamous cell carcinoma); CT chemotherapy; CCRT concurrent chemoradiotherapy; ROC the receiver operating characteristic.

$P$ denotes $P$ value for statistical significance based on $Z$ test; $P_{\mathrm{h}}$ denotes $P$ value for heterogeneity based on $\mathrm{Q}$ test. HR hazard ratio; CI confidence interval; NA not available. 
Table 2: Pooled and subgroup analyses of the main results for the meta-analysis of disease-free survival

\begin{tabular}{|c|c|c|c|c|c|c|c|}
\hline \multirow{2}{*}{ Categories } & \multirow{2}{*}{$n$} & \multirow{2}{*}{ Model } & \multirow{2}{*}{ HR (95\% CI) } & \multirow{2}{*}{$Z$} & \multirow{2}{*}{$\boldsymbol{P}$} & \multicolumn{2}{|c|}{ Heterogeneity } \\
\hline & & & & & & $P^{2}$ & $P_{\mathrm{h}}$ \\
\hline Disease-free survival (DFS) & $6(2904)$ & Random & $1.80(1.32-2.44)$ & 3.72 & $<0.001$ & $56.8 \%$ & 0.041 \\
\hline \multicolumn{8}{|l|}{ Study region } \\
\hline China & $3(2271)$ & Random & $1.60(1.08-2.36)$ & 2.36 & 0.018 & $52.9 \%$ & 0.119 \\
\hline Japan & $3(633)$ & Random & $2.10(1.23-3.59)$ & 2.72 & 0.007 & $59.8 \%$ & 0.083 \\
\hline \multicolumn{8}{|l|}{ Primary treatment } \\
\hline Surgery & $4(1196)$ & Fixed & $1.79(1.37-2.34)$ & 4.30 & $<0.001$ & $0.0 \%$ & 0.812 \\
\hline Others & $2(1708)$ & Random & $1.37(1.09-1.73)$ & 2.67 & 0.008 & $88.1 \%$ & 0.004 \\
\hline \multicolumn{8}{|l|}{ Clinical stage } \\
\hline All & $4(2384)$ & Fixed & $1.43(1.19-1.73)$ & 3.73 & $<0.001$ & $31.7 \%$ & 0.222 \\
\hline Others & $2(520)$ & Random & $2.70(1.13-6.40)$ & 2.25 & 0.025 & $66.7 \%$ & 0.083 \\
\hline \multicolumn{8}{|l|}{ Sample size } \\
\hline$<200$ & $3(378)$ & Random & $2.18(1.24-3.81)$ & 2.72 & 0.006 & $59.6 \%$ & 0.084 \\
\hline$\geq 200$ & $3(2526)$ & Fixed & $1.40(1.14-1.73)$ & 3.22 & 0.001 & $49.7 \%$ & 0.137 \\
\hline \multicolumn{8}{|l|}{ Cut-off value } \\
\hline$<0.1$ & $5(2768)$ & Fixed & $1.46(1.23-1.75)$ & 4.19 & $<0.001$ & $20.2 \%$ & 0.286 \\
\hline$\geq 0.1$ & $1(136)$ & Random & $4.30(1.94-10.13)$ & 3.53 & $<0.001$ & NA & NA \\
\hline
\end{tabular}

$P$ denotes $P$ value for statistical significance based on $Z$ test; $P_{\mathrm{h}}$ denotes $P$ value for heterogeneity based on $Q$ test. HR hazard ratio; CI confidence interval; NA not available.

Table 3: Summary of the association of C-reactive protein/albumin ratio and clinopathological parameters in solid tumors

\begin{tabular}{|c|c|c|c|c|c|c|c|}
\hline \multirow{2}{*}{ Category } & \multirow{2}{*}{$n$} & \multirow{2}{*}{ Model } & \multirow{2}{*}{ OR $(95 \% \mathrm{CI})$} & \multirow{2}{*}{$\boldsymbol{Z}$} & \multirow{2}{*}{$\boldsymbol{P}$} & \multicolumn{2}{|c|}{ Heterogeneity } \\
\hline & & & & & & $I^{2}$ & $P_{h}$ \\
\hline Histologic grade (poor vs. well or moderate) & $7(1983)$ & Fixed & $1.47(1.14-1.90)$ & 2.97 & 0.003 & $0.0 \%$ & 0.654 \\
\hline Clinical stage (III or IV vs. I or II) & $12(6279)$ & Random & $3.20(2.37-4.32)$ & 7.58 & $<0.001$ & $71.8 \%$ & $<0.001$ \\
\hline Tumor depth (T3+T4 vs. T1+T2) & $7(4367)$ & Random & $2.57(1.52-4.34)$ & 3.52 & $<0.001$ & $80.0 \%$ & $<0.001$ \\
\hline Lymph node metastasis (positive vs. negative) & $8(4496)$ & Random & $2.25(1.62-3.14)$ & 4.83 & $<0.001$ & $60.5 \%$ & 0.013 \\
\hline Distant metastasis (positive vs. negative) & $4(1266)$ & Random & $3.97(1.56-10.09)$ & 2.89 & 0.004 & $75.8 \%$ & 0.006 \\
\hline Smoking status (ever vs. never) & $5(3761)$ & Fixed & $1.16(1.00-1.34)$ & 1.95 & 0.052 & $45.9 \%$ & 0.117 \\
\hline
\end{tabular}

$P$ denotes $P$ value for statistical significance based on $Z$ test; $P_{\mathrm{h}}$ denotes $P$ value for heterogeneity based on $Q$ test. OR odds ratio; CI confidence interval.

criteria, all cohort studies had scores of six or more and were of high quality. The main characteristics of the included studies were listed in Supplementary Table 1.

\section{Association of CAR with OS}

The combined analysis of 23 studies with 10067 patients showed that patients with elevated CAR were expected to suffer unfavorable OS $(\mathrm{HR}=1.95,95 \% \mathrm{CI}$ : 1.70-2.25, $P<0.001$, random effects; Figure 2). When stratified by cancer type, high CAR predicted poor OS for patients with $\mathrm{PC}(\mathrm{HR}=2.25,95 \% \mathrm{CI}: 1.52-3.34$, $P<0.001$, random effects), CRC (HR $=2.26,95 \% \mathrm{CI}$ : 1.71-2.99, $P<0.001$, fixed effects), NPC (HR $=1.50$, 95\% CI: 1.29-1.74, $P<0.001$, random effects), ESCC $(\mathrm{HR}=1.84,95 \% \mathrm{CI}: 1.06-3.20, P=0.030$, random 
effects), $\mathrm{GC}(\mathrm{HR}=1.73,95 \% \mathrm{CI}: 1.31-2.29, P<0.001$, fixed effects $), \mathrm{HCC}(\mathrm{HR}=2.73,95 \% \mathrm{CI}: 2.07-3.60, P$ $<0.001$, fixed effects), head and neck cancer (including LSCC and OSCC $)(\mathrm{HR}=3.49,95 \% \mathrm{CI}: 1.78-6.85, P<$ 0.001 , fixed effects), and other cancers ( $\mathrm{HR}=1.41,95 \%$ CI: $1.24-1.60, P<0.001$, fixed effects). Similarly, when grouped based on study region, the prognostic role of elevated CAR in predicting shorter OS was obvious in studies originated from China $(\mathrm{HR}=1.84,95 \% \mathrm{CI}$ : $1.57-$ 2.17, $P<0.001$, random effects), Japan $(\mathrm{HR}=1.82,95 \%$ CI: $1.60-1.85, P<0.001$, fixed effects), and Korea (HR $=2.39,95 \% \mathrm{CI}: 1.34-4.24, P=0.003$, random effects). Moreover, the significant association of high CAR and worse OS did not change regardless of the subgroup analyses of primary treatment, clinical stage, cut-off selection, sample size, and cut-off value (Table 1).

The result of heterogeneity analysis illustrated that all of the included datasets of OS had extreme heterogeneity $\left(\mathrm{I}^{2}=68.9 \%, P_{\mathrm{h}}<0.001\right)$. Thus, we used a random-effects model to estimate the overall HR for OS. When the subgroup analysis was conducted to assess the source of heterogeneity based on study region, cancer type, primary treatment, clinical stage, sample size, and cutoff value, the heterogeneity was slightly reduced in most subgroups, but remained statistically significant in several subgroups (Table 1).

\section{Association of CAR with DFS}

Six studies comprising 2904 patients reported the outcomes for DFS, and the pooled result indicated that elevated CAR was associated with poor DFS $(\mathrm{HR}=1.80$, 95\% CI: 1.32-2.44, $P<0.001$, random effects; Figure $3)$. This significant associations were also found in the subgroup analyses of China $(\mathrm{HR}=1.60,95 \% \mathrm{CI}$ : 1.08 $2.36, P=0.018$, random effects), and Japan ( $\mathrm{HR}=2.10$, $95 \%$ CI: $1.23-3.59, P=0.007$, random effects). Similarly, the positive results were observed in the subgroup analyses based on primary treatment, clinical stage, sample size, and cut-off value (Table 2).

An extreme heterogeneity was also found among the included studies of DFS $\left(\mathrm{I}^{2}=56.8 \%, P_{\mathrm{h}}=0.041\right)$; thus, a random-effects model was applied to calculated the pooled outcome. When the subgroup analysis was conducted, the heterogeneity was obvious to be still significantly evident in most subgroups (Table 2).

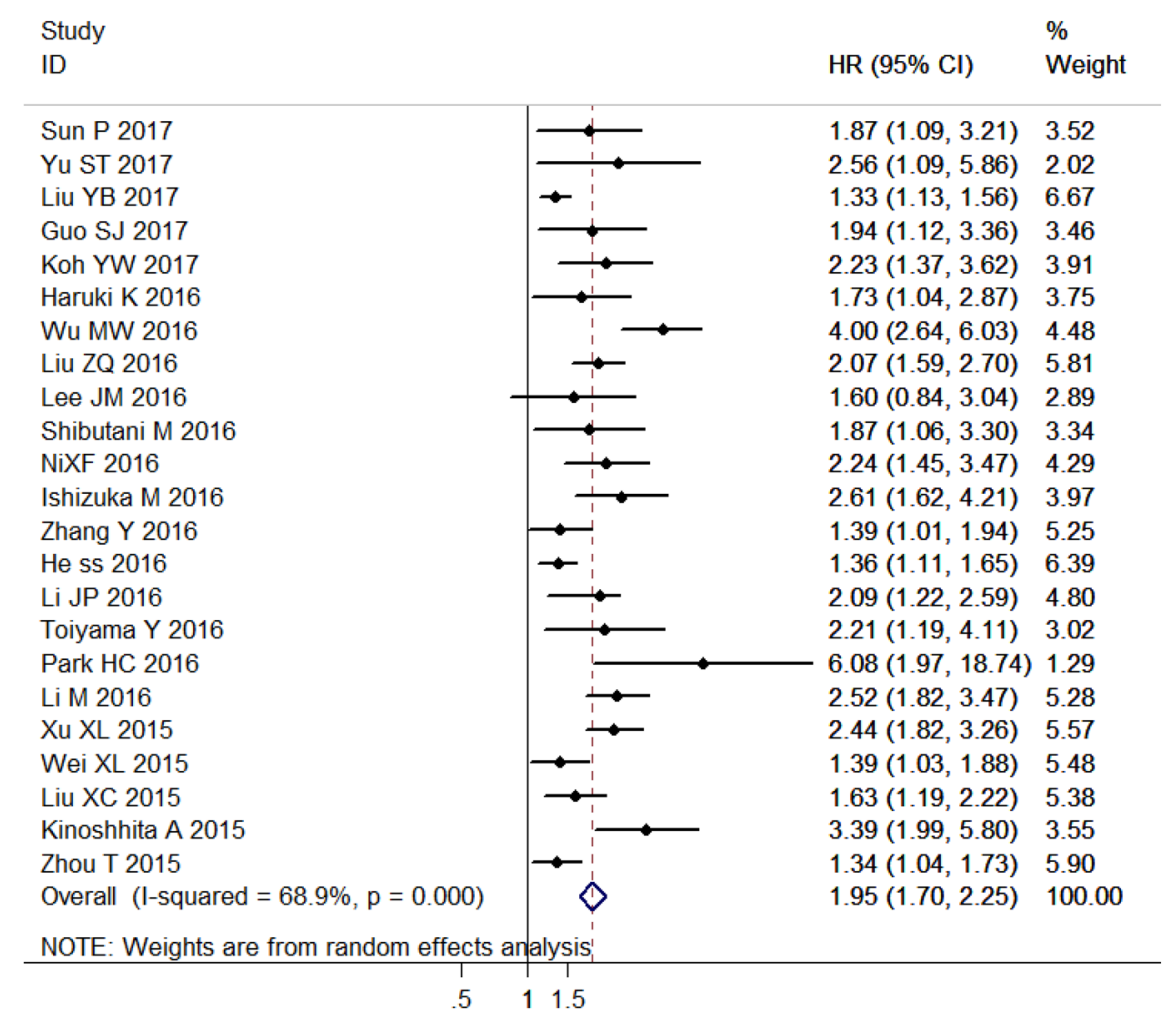

Figure 2: Forest plots of the association between C-reactive protein/albumin ratio and overall survival in patients with solid tumors. Abbreviation: HR hazard ratio; CI confidence interval. 


\section{Association of CAR with clinicopathological characteristics}

The relationship of CAR with clinicopathological features are illustrated in Table 3. Elevated CAR was correlated with certain phenotypes of tumor aggressiveness, such as poor histological grade (pooled odds ratio $[\mathrm{OR}]=1.47 ; 95 \% \mathrm{CI}=1.14-1.90 ; P=0.003$; fixed effects), serious clinical stage (pooled $\mathrm{OR}=3.20$; $95 \% \mathrm{CI}=2.37-4.32 ; \mathrm{P}<0.001 ;$ random effects), advanced tumor depth (pooled $\mathrm{OR}=2.57 ; 95 \% \mathrm{CI}=1.52-4.34$; $\mathrm{P}$ $<0.001$; random effects), positive lymph node metastasis (pooled $\mathrm{OR}=2.225 ; 95 \% \mathrm{CI}=1.62-3.14 ; \mathrm{P}<0.001$; random effects), and positive distant metastasis (pooled $\mathrm{OR}=3.97 ; 95 \% \mathrm{CI}=1.56-10.09 ; \mathrm{P}<0.001 ;$ random effects). However, no association existed between CAR and smoking status (pooled $\mathrm{OR}=1.16 ; 95 \% \mathrm{CI}=0.99$ $1.34 ; \mathrm{P}=0.052$; fixed effects).

\section{Sensitivity analysis and meta-regression analysis}

Sensitivity analysis was conducted to evaluate the robustness of association between CAR and survival outcomes, and the results suggested that no individual study significantly changed the overall HRs of our metaanalysis for OS and DFS (Figure 4).

We conducted meta-regression analysis to investigate the potential source of heterogeneity among studies for OS. In multivariate analysis, the results showed that study region $(P=0.387)$, cancer type $(P=0.710)$, primary treatment $(P=0.610)$, clinical stage $(P=0.234)$, sample size $(P=0.533)$, cut-off selection $(0.666)$, and cut- off value $(P=0.498)$ did not contribute to the source of heterogeneity for OS. Due to the small number of included studies, we did not perform meta-regression analysis to explore the underlying source of heterogeneity among studies concerning DFS.

\section{Publication bias}

Both Begg's and Egger's tests suggested a significant publication bias with regard to the pooled outcome of OS (Begg's test: $P=0.098$; Egger's test: $P$ $=0.001)$, and the funnel plot showed a certain degree of apparent asymmetry (Figure 5A), indicating potential publication bias. The trim-and-fill analysis showed that one more studies were needed to balance the funnel plot (Figure 5B). The adjusted $\mathrm{HR}$ and $95 \% \mathrm{CI}$ were attenuated but remains significant (pooled $\mathrm{HR}=1.93 ; 95 \% \mathrm{CI}=$ 1.67-2.22; $P<0.001$; random effects). Similarly, an obvious publication bias was also found concerning the pooled result of DFS (Begg's test: $P=0.060$; Egger's test: $P=0.005$; Figure 5C), and the HR estimate adjusted by the trim-and-fill analysis was still statistically significant after adding three unpublished studies (pooled $\mathrm{HR}=1.41$; $95 \% \mathrm{CI}=1.03-1.93 ; P=0.035$; random effects; Figure $5 \mathrm{D})$, thereby suggesting that the potential publication bias had minimal impact on the overall outcomes.

\section{DISCUSSION}

Although a first qualitative analysis of CAR levels related to prognostic outcome of various solid tumors has been published by Li et al. [20], there are still some

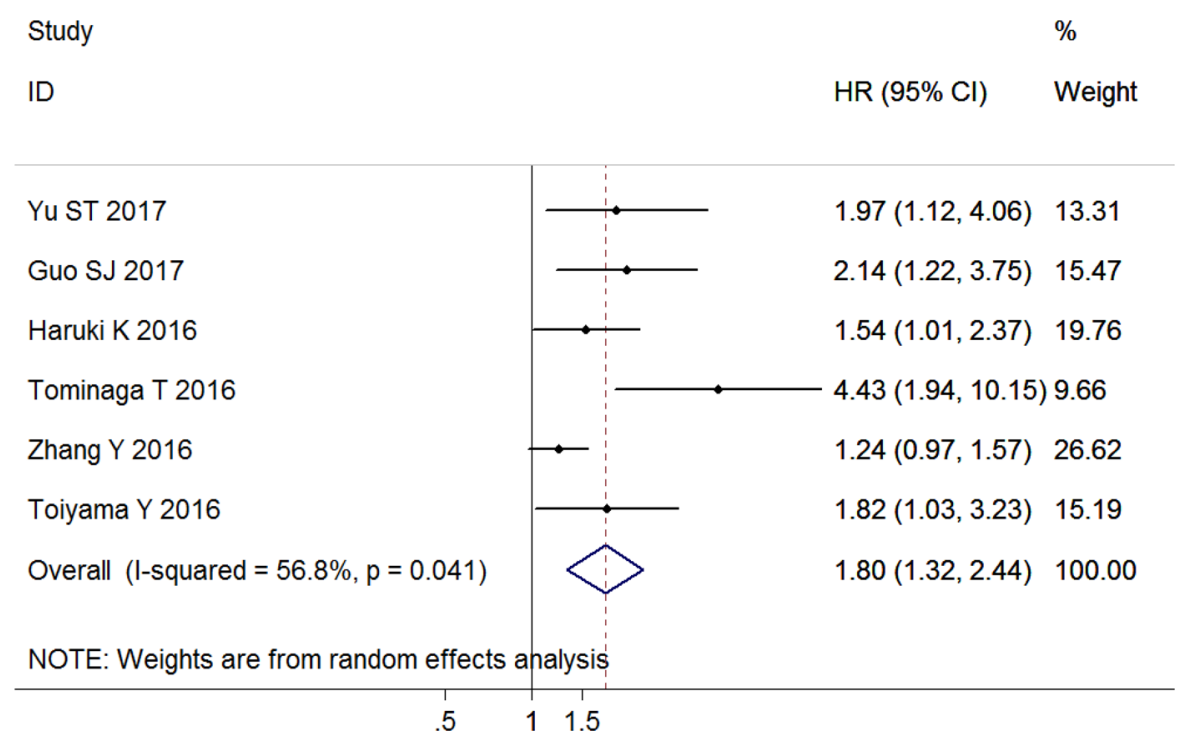

Figure 3: Forest plots of the association between C-reactive protein/albumin ratio and disease-free survival in patients with solid tumors. Abbreviation: HR hazard ratio; CI confidence interval. 
shortcomings that may negatively impact the reliability of the final results. Firstly, only 10 studies and 4592 patients were included in the previous meta-analysis, the numbers of which were extremely small and the evidence-based power was not strong enough. Secondly, both the HRs of univariate and multivariate analyses were provided in the report of $\mathrm{Wu}$ et al. [27], but only the data of univariate analysis was retrieved in the previous meta-analysis, which might introduce some imprecision because it has been not adjusted by the confounding factors. Thirdly, previous study only reported the pooled result of OS, but not the overall outcome of DFS, which was also an important survival endpoint of malignant tumors. Finally, the data synthesis by Li et al. [20] failed to explore the relationship between CAR concentration and clinical characteristics, which might lead to lose some valuable information. Therefore, it is necessary to perform an updated metaanalysis to further investigate the relationship between CAR and prognosis in patients with solid tumors.

Our meta-analysis comprised 24 studies with 10203 patients, and the synthesized data suggested that elevated pretreatment CAR predicted unfavorable prognosis, including OS and DFS, in patients with solid tumors. In the subgroup analyses, the significant association between elevated CAR and poor survival did not observably changed. Thus, CAR could be an independent predictor of all-caused and disease-specific mortality in solid cancers. However, these overall results were along with extreme heterogeneity which might affect the analysis, interpretation, and conclusions of this study. Different baseline characteristic of individual studies, such as study region, cancer type, primary treatment, clinical stage, cutoff selection, sample size, and cut-off value, might lead to inter-study heterogeneity. Meta-regression analysis was performed by using the abovementioned factors to further explore the source of heterogeneity, but none of them could complain the heterogeneity. Instead, we

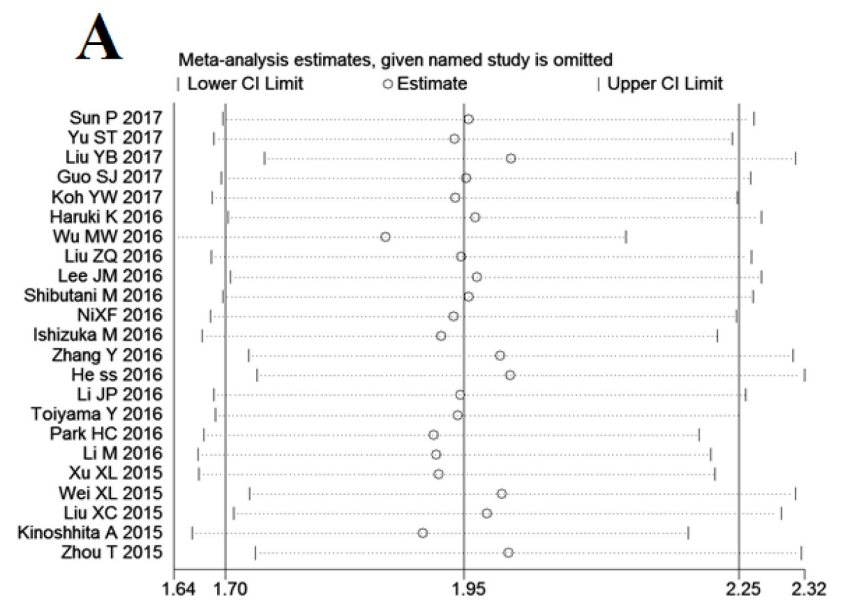

used a random-effect model to minimize the effect of the heterogeneity on OS and DFS. Moreover, despite the broad search criteria, evidences of publication bias were found among the studies concerning OS and DFS, which might have inflated the pooled results. After recalculated by trim-and-fill analyses, the adjusted pooled effect size remained statistically. This finding indicated that the publication bias may not have a systematic influence on the pooled outcomes, and further confirmed the reliability of our results.

To further explore the prognostic impact of CAR on solid cancers, we analyzed the correlation between CAR and clinicopathological features. According to the pooled outcomes, high levels of CAR was significantly associated with several features of tumor progression, including poor histological grade, serious clinical stage, advanced tumor depth, positive lymph node metastasis, and positive distant metastasis. These results strongly support the predictive value of elevated CAR on worse prognosis in malignances, and high CAR is closely related to more aggressive tumor behavior. Therefore, cancer patients with above clinical features would be probably to benefit more from CAR estimation for clinical-decisions making.

Similar to CAR, the GPS/mGPS system are based on the serum levels of two acute phase proteins, CRP and ALB, but there are several fundamental differences between them. First, the GPS/mGPS system is an evaluation based on a three-point score $(0,1$, or 2$)$, making it have a qualitative nature with discontinuous values, while CAR is a simple ratio, deemed as a quantitative variable with a continuous value. Thus, CAR could provide more information than the GPS/mGPS system. Second, the GPS/mGPS system may overestimate or underestimate the inflammatory or nutritional level in body because it separately scores the serum levels of CRP and ALB, whereas CAR can decrease the potential risk of overestimated or underestimated results for the

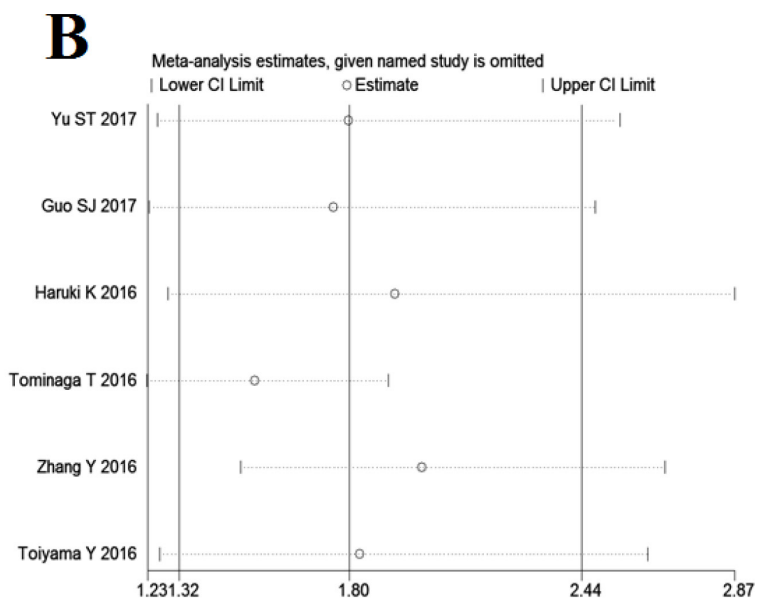

Figure 4: Effect of individual studies on pooled hazard ratios (HR) for the relationship between C-reactive protein/ albumin ratio and prognosis of solid tumors. (A) Sensitivity analysis for overall survival. (B) Sensitivity analysis for disease-free survival. 
reason that it synthesize of the CRP and ALB values more reasonable $[28,43]$. Third, as a continuous score, CAR has the ability to further distinguish the patients with the same score according to the GPS/mGPS system [27]. On the other hand, Liu et al. [23] reported that CAR, GPS, and mGPS were all predictors of survival in ovarian cancer by univariate analysis, but only CAR remained as an independent index for prognosis after adjusted by multivariate analysis, the result of which was the same to some reports on PC [26], NPC [36], and HCC [43]. Meanwhile, AUC analysis further identified the better predictive accuracy of CAR rather than other inflammation-based prognostic scores, including GPS and mGPS, which is consistent with several previous studies in CRC [33], ESCC [40, 41], GC [42] and HCC [43]. Therefore, the GPS/mGPS system exposed some defects in its clinical practice, and it can be said that CAR is superior to the GPS/mGPS system for prognostic prediction.

CRP is a representative acute phase response protein whose levels rapidly increase in response to inflammation, and has been regarded as a definitive marker of systemic inflammation in vivo [45]. In clinical practice, it has been a most widely used index to evaluate the severity of the systemic inflammation or outcomes of various inflammation- related disorders. Approximate $90 \%$ of normal populations have a serum CRP concentration of less than $0.3 \mathrm{mg} / \mathrm{dl}$, while cancer patients represent a significantly higher serum CRP level. CRP is mainly synthesized by liver and is strongly induced by proinflammatory cytokines, especially interleukin-6 (IL-6). Tumor tissue can trigger inflammatory response, further promotes the accumulation of inflammatory cells and the release of pro-inflammatory cytokines, resulting in an increased production of CRP by hepatocytes [46]. Simultaneously, some tumor cells, including ESCC and NSCLC, also have the ability to secret CRP alone and may contribute to the serum CRP level [47]. Therefore, cancers often occur along with increased CRP levels. Elevated CRP levels may contribute to create a favorable microenvironment for tumor cells proliferation and metastatization. Numerous epidemiological studies have showed that elevated CRP has been correlated with persistent fatigue, increased weight loss, low performance status and poor survival of cancer patients [48]. With the presence of elevated serum CRP concentration, serum level of vascular endothelial growth factor is also increased, which plays a critical role in promoting cancer angiogenesis and improving oxygen supply of cancer cells [49]. Moreover, CRP genetic polymorphism was associated with lymph node (LN) metastasis in several cancer types, including ESCC, NSCLC, and breast cancer [50]. The CRP 1846C $>$ T polymorphism could be a novel predictor of LN involvement in ESCC, which was more accurate than computed tomography [51]. These findings indicated that CRP not only exists as an inflammatory marker, but also act as a prognostic predictor of malignant tumors.

ALB is also produced by the liver, which plays an important role in the maintenance of intravascular oncotic pressure, the transport of substances and the scavenging of free radicals. As one of the most common markers for assessing nutritional status, the serum ALB levels fall sharply during cancer progression because both malnutrition and systematic inflammation can suppress
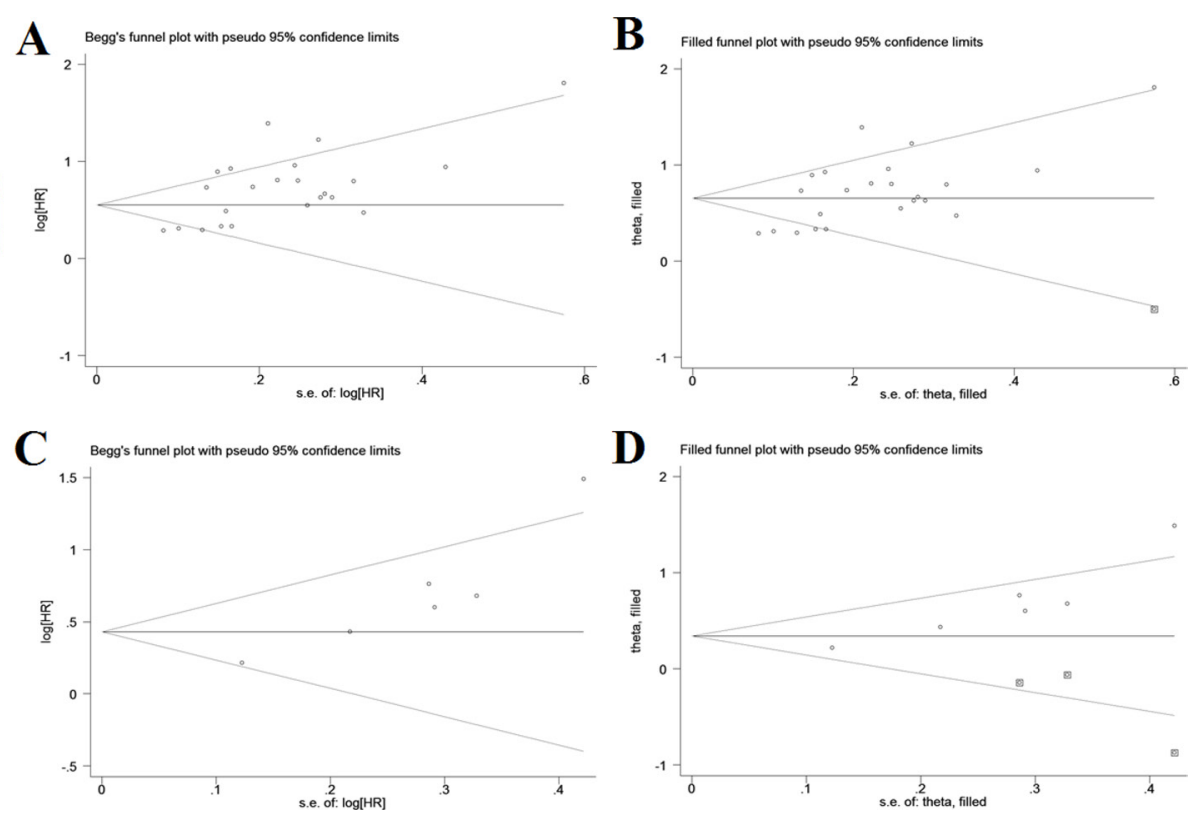

Figure 5: Funnel plots for assessment of potential publication bias in studies of C-reactive protein/albumin ratio and prognosis of solid tumors. (A) Funnel plot of publication bias for studies reporting overall survival. (B) Funnel plot adjusted with trim and fill method for studies reporting overall survival. (C) Funnel plot of publication bias for studies reporting disease-free survival. (D) Funnel plot adjusted with trim and fill method for studies reporting disease-free survival. 
ALB synthesis [52]. During the malignant progress of tumors, tumor-related inflammatory cells are activated and secret several pro-inflammatory cytokines, including tumor necrosis factor, IL-1, IL-6, and IL-8. These cytokines can inhibit the ability of liver cells to generate ALB and increases the permeability of capillaries, which lead to a direct decrease of ALB concentration in the circulatory system [53]. Moreover, the development of micrometastases in liver tissue induces liver dysfunction and reduces the synthesis of ALB [54]. Thus, numerous studies have suggested that pretreatment serum ALB can be an effective prognostic indicator for cancer patients, as the low level of serum ALB predicts the worse prognosis.

Although both pretreatment CRP and ALB can be the independent prognostic factors of cancer patients, they are not perfect predictors because they can be easily influenced by some nontumor-related factors, such as diet, overhydration, and inflammation outside the tumor site. Several researches have revealed that the occurrence of decreased serum ALB levels, namely hypoalbuminemia, is secondary to the elevation of serum CRP, as many cancer patients with hypoalbuminemia already have high concentrations of serum CRP [55]. So CRP and ALB, represent two complementary statuses in vivo, are more appropriate to be combined as a composite parameter rather than being used as single predictors for prognosis of solid tumors.

Similar to other systematic review and metaanalysis, some limitations of this meta-analysis should be acknowledged. First, because the numbers of subgroup analyses dealing with each cancer type were less than 5 , the evidence supporting the findings of the particular carcinomas might be less powerful. Moreover, due to the limited number of studies, we were unable to conduct subgroup analyses for certain types of cancer, such as OC, RCC, NSCLC and SCLC. Second, because no acknowledged threshold was available, different cutoffs to define "high" level of CAR were used in our included studies, which might also lead to clinical and statistical heterogeneity, and affect the availability of CAR as a predictive index in cancer prognosis to some degree. Third, the fact that researches with positive results tended to be published than works with null or negative outcomes, might induce reporting bias and potentially exaggerate the association between CAR and prognosis in solid tumors. Fourth, as all included papers were of East Asian origin, there may limitation on racial representation and the conclusions should be taken cautiously for other ethnic populations. Fifth, our meta-analysis primarily concentrated on pretreatment CAR, and the clinical significance of posttreatment CAR change, which may dynamic reflect the variation of the balance between host inflammatory response and nutritional status after therapy, is completed unclear. Finally, only evidence for correlation study was provided by the present meta-analysis, which could not be simply interpreted as causal relationship.
In conclusion, our meta-analysis suggested that elevated CAR is closely associated with poor survival including OS and DFS, as well some unfavorable clnilcopathological features in patients with solid tumors. Given its quickness, convenience, inexpensiveness, and reproducibility in clinical application, we believe that CAR can serve as a useful, effective index for the algorithm concerning the prognostic evaluation of cancer patients. However, due to the limitations of our metaanalysis, larger well-designed studies are recommended to validate the prognostic value of CAR in various tumors, and further explore the superiority of CAR over other traditional inflammatory markers by head to head comparisons.

\section{MATERIALS AND METHODS}

\section{Literature search strategy}

This meta-analysis was conducted according to the guidelines of the Preferred Reporting Items for Systematic Reviews and Meta-Analyses (PRISMA) [56]. A comprehensive electronic searching of PubMed, Embase, Web of Science, Cochrane Library databases, and China National Knowledge Infrastructure was conducted without language restrictions. We used combinations of the following key words: "C-reactive protein/albumin ratio or $\mathrm{C}$ reactive protein to albumin ratio or $\mathrm{C}$-reactive albumin ratio or $\mathrm{CRP} / \mathrm{Alb}$ ratio or CAR (all fields), cancer or tumor or malignancy or neoplasm or carcinoma (all fields), and prognosis or prognostic or survival or outcome (all fields)". The final search was updated on Augest 2017. The citation lists of the included studies in this metaanalysis as well as any appropriate review articles were also screened for further relevant papers.

\section{Study selection criteria}

Publications were eligible for this meta-analysis if they met all of the following criteria: (1) full papers with cohort design reported the relationship between pretreatment CAR and prognostic outcomes of solid tumors, such as OS, and DFS; (2) the patients with solid tumors were divided into two groups according to the CAR level, regardless of the cut-off value; (3) the HRs and 95\% CIs for survival outcomes could be directly obtained from the original data or indirectly calculated from sufficient information. Studies were considered ineligible if they were reviews, conference abstracts, editorials, or letters, and they belonged to basic research and animal experiments. For multiple publications from the same authors or institutes with the same patient cohorts, only the most recent data were retained in the final analysis to avoid duplicate information. 


\section{Data extraction and quality assessment}

Two authors (JYW and WKT) independently reviewed the included studies and performed data extraction. Any disagreement between the reviewers was resolved by consensus. The following information was recorded for each study: first name of the authors, year of publication, study region, cancer type, primary treatment, duration period, follow-up time, sample number, cutoff selection, cut-off value, elevated CAR case number, clinical features, survival outcomes, HR estimation, and quality scores. The HRs and the corresponding 95\% CIs were extracted from multivariate analyses where available, because it is more precise due to accounting for the confounding factors. Otherwise, HRs were extracted from the univariate analysis or calculated using the KaplanMeier survival curves [57].

The quality of each study was assessed by Newcattle-Ottawa Scale (NOS) according to the following categories: selection (four points), comparability (two points), and outcome of interest (three points) [58]. The total score of NOS ranged from 0 to 9 , and we considered studies of six or more as high quality.

\section{Statistical analysis}

All statistical analyses were performed by using STATA version 13.0 (STATA Corporation, College Station, TX, USA). Combined HRs and their 95\% CIs were used to measure the impact of elevated CAR on survival. An observed HR greater than 1 indicated poor prognosis for patients with high CAR with its $95 \%$ CI exceeding 1 . The statistical significance of the pooled HR was determined through $Z$-test, and $P<0.05$ indicated statistical significance. Subgroup analyses were conducted according to cancer type (at least two trials must report the same outcome for the same cancer type; otherwise, they will be assigned to a subgroup designated "Others"), study region ("China", "Japan" and "Korea"), primary treatment ("surgery", "CT", "CCRT" and "none reported [NR]"), clinical stage ("All stages", "I-III", and "advanced"), cutoff selection ("ROC", "cutoff finder", and "median"), and sample size (" $<200$ " and " $\geq 200$ "). Moreover, a subgroup analysis based on cutoff values were also performed, namely " $<0.1$ " and " $\geq 0.1$ ", because 0.1 was the median of the cut-off values to define "high" level of CAR among the included studies. Meta-regression analysis was also performed to determine the potential sources of heterogeneity. The ORs and the corresponding 95\% CIs were used to estimate the correlation between CAR and clinicopathological features. All statistical tests were two sided.

Statistical heterogeneity was examined by the Cochran's Q statistic qualitatively, and the $\mathrm{I}^{2}$ metric quantitatively $\left(\mathrm{I}^{2} \leq 25 \%\right.$, no heterogeneity; $25 \%<\mathrm{I}^{2}<$ $50 \%$, moderate heterogeneity; and $\mathrm{I}^{2} \geq 50 \%$, extreme heterogeneity) [59]. When significant heterogeneity had been observed among the studies $\left(P<0.10\right.$ or $\left.\mathrm{I}^{2}>50 \%\right)$, the pooled HR estimation of each study was calculated using a random-effects model (DerSimonian and Laird method). Otherwise, a fixed-effects model was applied (Mantel-Haenszel method) [60]. Sensitivity analysis was conducted by sequentially omitting each individual study to validate the stability of the meta-analysis outcomes. The effect of potential publication bias on the outcomes was quantitatively evaluated through Begg's and Egger's asymmetry tests [61], and was visually evaluated using funnel plots. If significant publication bias existed, trim and fill method was performed to validate the robust of the meta-analysis results [62]. The statistical significance of Begg's and Egger's tests was defined as $P<0.10$.

\section{CONFLICTS OF INTEREST}

The authors declared no conflicts of interests.

\section{REFERENCES}

1. Coussens LM, Werb Z. Inflammation and cancer. Nature. 2002; 6917:860-867.

2. Zhang Q, Zhu B, Li Y. Resolution of cancer-promoting inflammation: a new approach for anticancer therapy. Front Immunol. 2017; 8:71.

3. Plummer M, de Martel C, Vignat J, Ferlay J, Bray F, Franceschi S. Global burden of cancers attributable to infections in 2012: a synthetic analysis. Lancet Glob Health. 2016; 4:e609-616.

4. Lee G, Walser TC, Dubinett SM. Chronic inflammation, chronic obstructive pulmonary disease, and lung cancer. Current Opin Pulm Med. 2009; 15:303-307.

5. Deivendran S, Marzook KH, Radhakrishna Pillai M. The role of inflammation in cervical cancer. Adv Exp Med Biol. 2014; 816:377-399.

6. Wessler S, Krisch LM, Elmer DP, Aberger F. From inflammation to gastric cancer - the importance of Hedgehog/GLI signaling in Helicobacter pylori-induced chronic inflammatoty and neoplastic diseases. Cell Commun Signal. 2017; 15:15.

7. Gonzale-Trejo S, Carrillo JF, Carmona-Herrera DD, BazGutierrez P, Herrera-Goepfert R, Nunez G, Ochoa-Carrillo FJ, Gallardo-Rincon D, Aiello-Crocifiglio V, Onate-Ocana LF. Baseline serum albumin and other common clinical markers are prognostic factors in colorectal carcinoma: a retrospective cohort study. Medicine (Baltimore). 2017; 96:e6610.

8. Xie FL, Wang YQ, Peng LF, Lin FY, He YL, Jiang ZQ. Beneficial effect of educational and nutritional intervention on the nutritional status and compliance of gastric cancer patients undergoing chemotherapy: a randomized trial. Nutr Cancer. 2017; 69:762-771.

9. Cox S, Powell C, Carter B, Hurt C, Mukherjee S, Crosby TD. Role of nutritional status and intervention in oesophageal 
cancer treated with definitive chemoradiotherapy: outcomes from SCOPE1. Br J Cancer. 2016; 115:172-177.

10. Sun H, He B, Nie Z, Pan Y, Lin K, Peng H, Xu T, Chen X, $\mathrm{Hu} \mathrm{X}, \mathrm{Wu} \mathrm{Z}$, Wu D, Wang S. A nomogram based on serum bilirubin and albumin levels predicts survival in gastric cancer patients. Oncotarget. 2017; 8:41305-41318. http:// doi.org/10.18632/oncotarget.17181.

11. Dikken JL, van de Velde CJ, Gonen M, Verheij M, Brennan MF, Coit DG. The New American Joint Committee on Cancer/International Union Against Cancer staging system for adenocarcinoma of the stomach: increased complexity without clear improvement in predictive accuracy. Ann Surg Oncol. 2012; 19:2443-2451.

12. Lee JY, Kim HI, Kim YN, Hong JH, Alshomimi S, An JY, Cheong JH, Hyung WJ, Noh SH, Kim CB. Clinical significance of the Prognostic Nutritional Index for predicting short- and long-term surgical outcomes after gastroctomy: a retrospective analysis of 7781 gastric cancer patients. Medicine (Baltimore). 2016; 95:e3539.

13. Shrotriya S, Walsh D, Bennani-Baiti N, Thomas S, Lorton C. C-reactive protein is an important biomarker for prognosis tumor recurrence and treatment response in adult solid tumors: a systematic review. PLoS One. 2015; 10: e0143080.

14. Liu Y, He X, Pan J, Chen S, Wang L. Prognostic role of Glasgow prognostic score in patients with colorectal cancer: evidence from population studies. Sci Rep. 2017; 7:6144.

15. Dolan RD, McSorley ST, Horgan PG, Laird B, McMilan DC. The role of the systemic inflammatory response in predicting outcomes in patients with advanced inoperable cancer: systematic review and meta-analysis. Crit Rev Oncol Hematol. 2017; 116:134-146.

16. Zhong JH, Huang DH, Chen ZY. Prongostic role of systemic immune-inflammation index in solid tumors: a systematic review and meta-analysis. Oncotarget. 2017; 8:75381-75388. https://doi.org/10.18632/oncotarget.18856.

17. Liu BZ, Tao L, Chen YZ, Li XZ, Dong YL, Ma YJ, Li SG, Li F, Zhang WJ. Preoperative body mass index, blood albumin and triglycerides predict survival for patients with gastric cancer. PLoS One. 2016; 11:e0157401.

18. Ge X, Dai X, Ding C, Tian H, Yang J, Gong J, Zhu W, Li N, Li J. Early postoperative decrease of serum albumin predicts surgical outcome in patients undergoing colorectal resection. Dis Colon Rectum. 2017; 60:326-334.

19. Ranzani OT, Zampieri FG, Forte DN, Azevedo LC, Park M. C-reactive protein/albumin ratio predicts 90 -days mortality of septic patients. PLoS One. 2016; 8:e59321.

20. Li N, Tian GW, Wang Y, Zhang H, Wang ZH, Li G. Prognostic role of the pretreatment $\mathrm{C}$-reactive protein/albumin ratio in solid tumors: a meta-analysis. Sci Rep. 2017; 7:41298.

21. Sun P, Chen C, Xia Y, Bi X, Liu P, Zhang F, Yang H, An X, Jiang W, Wang F. The ratio of C-reactive protein/albumin is a novel inflammatory predictor of overall survival in cisplatinbased treated patients with metastatic nasopharyngeal carcinoma. Dis Markers. 2017; 2017:6570808.
22. Yu ST, Zhou Z, Cai Q, Liang F, Han P, Chen R, Huang XM. Prognostic value of the C-reactive protein/albumin ratio in patients with laryngeal squamous cell carcinoma. Onco Targets Ther. 2017; 10:879-884.

23. Liu Y, Chen S, Zheng C, Ding M, Zhang L, Wang L, Xie M, Zhou J. The prognostic value of the preoperative c-reactive protein/albumin ratio in ovarian cancer. BMC Cancer. 2017; 17:285.

24. Guo S, He X, Chen Q, Yang G, Yao K, Dong P, Ye Y, Chen D, Zhang Z, Qin Z, Liu Z, Xue Y, Zhang M, et al. The $\mathrm{C}$-reactive protein/albumin ratio, a validated prognostic score, predicts outcome of surgical renal cell carcinoma patients. BMC Cancer. 2017; 17:171

25. Koh YW, Lee HW. Prognostic impact of C-reactive protein/ albumin ratio on the overall survival of patients with advanced nonsmall cell lung cancers receiving palliative chemotherapy. Medicine (Baltimore). 2017; 96:e6848.

26. Haruki K, Shiba H, Shirai Y, Horiuchi T, Iwase R, Fujiwara Y, Furukawa K, Misawa T, Yanaga K. The C-reactive protein to albumin ratio predicts long-term outcomes in patients with pancreatic cancer after pancreatic resection. World J Surg. 2016; 40:2254-2260.

27. Wu M, Guo J, Guo L, Zuo Q. The C-reactive protein/albumin ratio predicts overall survival of patients with advanced pancreatic cancer. Tumor Biol. 2016; 37:12525-12533.

28. Liu Z, Jin K, Guo M, Long J, Liu L, Liu C, Xu J, Ni Q, Luo $\mathrm{G}, \mathrm{Yu} \mathrm{X}$. Prognostic value of the CRP/Alb ratio, a novel inflammation-based score in pancreatic cancer. Ann Surg Oncol. 2017; 24:561-568.

29. Lee JM, Lee HS, Hyun JJ, Choi HS, Kim ES, Keum B, Seo YS, Jeen YT, Chun HJ, Um SH, Kim CD. Prognostic value of inflammation-based markers in patients with pancreatic cancer administered gemcitabine and erlotinib. World J Gastrointest Oncol. 2016; 8:555-562.

30. Shibutani M, Maeda K, Nagahara H, Iseki Y, Hirakawa K, Ohira M. The significance of the C-reactive protein to albumin ratio as a marker for predicting survival and monitoring chemotherapeutic effectiveness in patients with unresectable metastatic colorectal cancer. Springerplus. 2016; 5:1787.

31. Ishizuka M, Nagata H, Takagi K, Iwasaki Y, Shibuya N, Kubota K. Clinical significance of the C-reactive protein to albumin ratio for survival after surgery for colorectal cancer. Ann Surg Oncol. 2016; 23:900-907.

32. Tominaga T, Nonaka T, Sumida Y, Hidaka S, Sawai T, Nagayasu $\mathrm{T}$. The C-reactive protein to albumin ratio as a predictor of severe side effects of adjuvant chemotherapy in stage III colorectal cancer patients. PLoS One. 2016; 11:e0167967.

33. Ni XF, Wu P, Wu J, Ji M, Shao YJ, Zhou WJ, Jiang JT, $\mathrm{Wu}$ CP. C-reactive protein/albumin ratio as a predictor of survival of metastatic colorectal cancer patients receiving chemotherapy. Int J Clin Exp Pathol. 2016; 9:5525-5534.

34. Zhang Y, Zhou GQ, Liu X, Chen L, Li WF, Tang LL, Liu Q, Sun Y, Ma J. Exploration and validation of C-reactive 
protein/albumin ratio as a novel inflammation-based prognostic marker in nasopharyngeal carcinoma. J Cancer. 2016; 7:1406-1412.

35. He S, Wang Y, Chen H, Yang L, Liang S, Lu L, Chen Y. C-reactive protein/albumin ratio (CAR) as a prognostic factor in patients with non-metastastatic nasopharyngeal carcinoma. J Cancer. 2016; 7:2360-2366.

36. Li JP, Chen SL, Liu XM, He X, Xing S, Liu YJ, Lin YH, Liu WL. A novel inflammation-based stage (I stage) predicts overall survival of patients with nasopharyngeal carcinoma. Int J Mol Sci. 2016; 17:1900.

37. Toiyama T, Shimura T, Yasuda H, Fujikawa H, Okita Y, Kabayashi M, Ohi M, Yoshiyama S, Hiro J, Araki T, Inoue Y, Mohri Y, Kusunoki M. Clinical burden of C-reactive protein/albumin ratio before curative surgery for patients with gastric cancer. Anticancer Res. 2016:36:6491-6498.

38. Park HC, Kim MY, Kim CH. C-reactive protein/albumin ratio as prognostic score in oral squamous cell carcinoma. $\mathrm{J}$ Korean Assoc Oral Maxilllofac Surg. 2016; 42:243-250.

39. Li M, Zhang P. Predictive value of C-reactive protein/ albumin ratio on prognosis of patients with primary hepatocellular carcinoma. Cancer Res Prev Treat. 2016; 43:598-601.

40. Xu XL, Yu HQ, Hu W, Song Q, Mao WM. A novel inflammation-based prognostic score, the C-reactive protein/albumin ratio predicts the prognosis of patients with operable esophageal squamous cell carcinoma. PLoS One. 2015; 10:e138657.

41. Wei XL, Wang FH, Zhang DS, Qiu MZ, Ren C, Jin Y, Zhou YX, Wang DS, He MM, Bai L, Wang F, Luo HY, Li YH, $\mathrm{Xu} \mathrm{RH}$. A novel inflammation-based prognostic score in esophageal squamous cell carcinoma: the C-reative protein/ albumin ratio. BMC Cancer. 2015; 15:350.

42. Liu X, Sun X, Liu J, Kong P, Chen S, Zhan Y, Xu D. Preoperative C-reactive protein/albumin ratio predicts prognosis of patients after curative resection for gastric cancer. Transl Oncol. 2015; 8:339-345.

43. Kinoshita A, Onoda H, Imai N, Iwaku A, Oishi M, Tanaka K, Fushiya N, Koike K, Nishino H, Mataushima M. The C-reactive protein/albumin ratio, a novel inflammationbased prognostic score, predicts outcomes in patients with hepatocellular carcinoma. Ann Surg Oncol. 2015; 22:803-810.

44. Zhou T, Zhan J, Hong S, Hu Z, Fang W, Qin T, Ma Y, Yang Y, He X, Zhao Y, Huang Y, Zhao H, Zhang L. Ratio of $\mathrm{C}$-reactive protein/albumin is an inflammatory prognostic score for predicting overall survival of patients with small cell lung cancer. Sci Rep. 2015; 5:10481.

45. Sciarra A, Gentilucci A, Salciccia S, Pierella F, Del Bianco F, Gentile V, Silvestri I, Cattarino S. Prognostic value of inflammation in prostate cancer progression and response to therapeutic: a critical review. J Inflamm. 2016; 13:35.

46. Asegaonkar SB, Asegaonkar BN, Takalkar UV, Advani S, Thorat AP. C-reactive protein and breast cancer: new insight from old molecule. Int J Breast Cancer. 2015; 2015:145647.

47. Amono K, Maeda I, Morita T, Baba M, Miura T, Hama T, Mori I, Nakajima N, Nishi T, Sakurai H, Shimoyama S, Shinjo T, Shirayama H, et al. C-reactive protein, symptoms and activity of daily living in patients with advanced cancer receiving palliative care. J Cachexia Sarcopenia Muscle. 2017; 8:457-465.

48. Thurner EM, Krenn-Pilko S, Langsenlehner U, Stojakovic T, Pichler M, Gerger A, Kapp KS, Langsenlehner T. The elevated C-reactive protein level is associated with poor prognosis in prostate cancer patients treated with radiotherapy. Eur j cancer. 2015; 51:610-9.

49. Li Z, Xue TQ, Chen XY. Predictive values of serum VEGF, CRP levels combined with contrast enhanced MRI in hepatocellular carcinoma patients after TACE. Am J Cancer Res. 2016; 6:2375-2385.

50. Kito M, Motoyama S, Fujita K, Miura M, Nanjo H, Sato N, Shimizu D, Sato T, Makino K, Sugawara T, Kato A, Tamura D, Takahashi K, et al. CRP 1846C > T genetic polymorphism is associated with lymph node metastasis and/or severe lymphatic invasion in endometrial cancer. Tohoku J Exp Med. 2015; 237:25-30.

51. Motoyama S, Mori K, Kamei T, Miura M, Hinai Y, Sato Y, Yoshino K, Sasaki T, Miyata G, Seta Y, Ogawa J. Evaluation of the risk of lymph node metastasis using CRP 1846C > T genetic polymorphism in submucosal thoracic esophageal squamous cell carcinoma. Ann Sur Oncol. 2013; 20:1978-1984.

52. Ataseven B, du Bois A, Reinthaller A, Traut A, Heitz F, Aust S, Prader S, Polterauer S, Harter P, Grimm C. Preoperative serum albumin is associated with post-operative complication rate and overall survival in patients with epithelial ovarian cancer undergoing cytoreductive surgery. Gynecol Oncol. 2015; 138:560-565.

53. Gupta D, Lis CG. Pretreatment serum albumin as a predictor of cancer survival: a systematic review of the epidemiological literature. Nutr J. 2010; 9:69.

54. Chen Z, Shao Y, Wang K, Cao W, Xiong Y, Wu R, Luo S, $\mathrm{Xu} \mathrm{X}, \mathrm{He} \mathrm{X}$. Prognostic role of pretreatment serum albumin in renal cell carcinoma: a systematic review and metaanalysis. Onco Targets Ther. 2016; 9:6701-6710.

55. Kostner AH, Kersten C, Lowenmark T, Ydsten KA, Peltonen R, Isoniemi H, Haglund C, Gunnarsson U, Isaksson $\mathrm{B}$. The prognostic role of systemic inflammation in patients with undergoing resection of colorectal liver metastases: C-reactive protein (CRP) is a strong negative prognostic biomarker. J Surg Oncol. 2016; 114:895-899.

56. Moher D, Shamseer L, Clarke M, Ghersi D, Liberati A, Petticrew M, Shekelle P, Stewart LA, and PRISMA-P Group. Preferred reporting items for systematic review and meta-analysis protocols (PRISMA-P) 2015 statement. Syst Rev. 2015; 4:1.

57. Parmar MK, Torri V, Stewart L. Extracting summary statistics to perform meta-analyses of the published literature for survival endpoints. Stat Med. 1998; 17:2815-34. 
58. Maxwell L, Santesso N, Tugwell PS, Wells GA, Judd M, Buchbinder R. Method guidelines for Cochrane Musculoskeletal Group systematic reviews. J Rheumatol. 2006; 33:2304-11.

59. Higgins JP, Thompson SG, Deeks JJ, Altman DG. Measuring inconsistency in meta- analyses. BMJ. 2003; 327:557-60.

60. Mantel N, Haenszel W. Statistical aspects of the analysis of data from retrospective studies of disease. J Natl Cancer Inst. $1959 ; 22: 719-48$.
61. Egger M, Davey SG, Schneider M, Minder C. Bias in metaanalysis detected by a simple, graphical test. BMJ. 1997; 315:629-34.

62. Peters JL, Sutton AJ, Jones DR, Abrams KR, Rushton L. Performance of the trim and fill method in the presence of publication bias and between-study heterogeneity. Stat Med. 2007; 26:4544-4562. 